\title{
Resource Dynamics Dictate Diversity
}

\section{A model that treats species and food sources as spins in a spin glass finds a surprising limit on species diversity for some types of ecosystems.}

\author{
By Marric Stephens
}

A central tenet of theoretical ecology holds that two species cannot exploit the same resource for long. IEventually, one species will dominate while the other either finds a new niche or goes extinct. This principle means that, in an ecosystem with self-replenishing food resources (plants or prey animals, for example), the maximum number of species is equal to the number of distinct resources that the ecosystem contains. Now, Wenping Cui at Boston University and colleagues have shown that when resources are externally supplied at a constant rate instead-as is the case for nutrients that feed gut bacteria-the cap on the number of species is cut by half. The finding could help to design experiments to study microbial diversity.

Theoretical ecology began a century ago with a pair of equations that describe predator-prey populations. Since then, models have become significantly more complex, but exactly how the species number depends on resource dynamics remains unclear. To address this issue, Cui and his colleagues turned to a model developed for spin glasses, but instead of

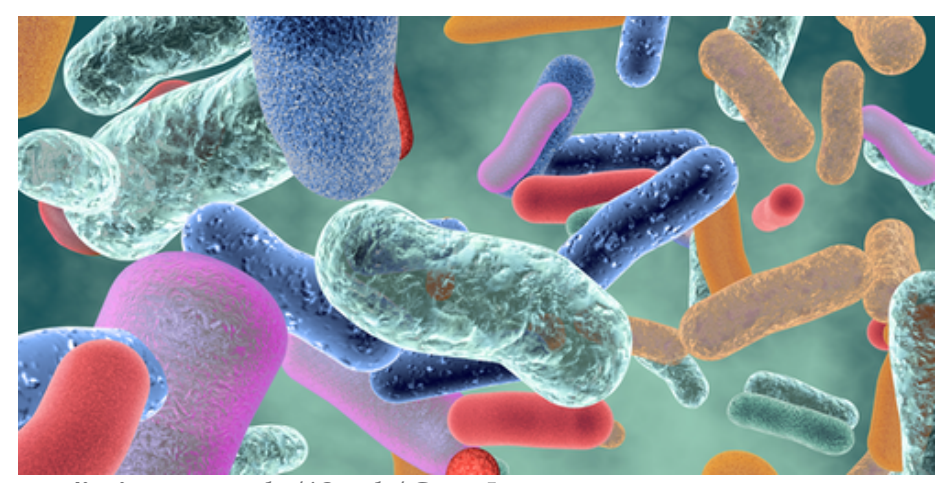

Credit: image ${ }_{j}$ ungle/iStock/GettyImages simulating the spins of a magnet, the team used the model to study the resources and consumers in an ecosystem.

The team's theory shows that when species' consumption preferences are random and overlapping, an ecosystem built on self-renewing resources can host the same number of species as there are food options. But in an ecosystem with externally supplied nutrients, resources distribute more narrowly-more resources go to the most flexible species-and the maximum number of species halves. The researchers say that their result, which they confirmed using numerical simulations, arises because of an increased level of competition between species.

This research is published in Physical Review Letters.

Marric Stephens is a Corresponding Editor for Physics based in Bristol, UK. 